\section{6 \\ SOCIAL AUTOPSY: A COMMUNITY BASED INTERVENTION IN PREVENTING ROAD TRAFFIC INJURIES-EXPERIENCE FROM BANGLADESH}

doi:10.1136/injuryprev-2012-040590u.6

${ }^{1,2}$ MK Baset, ${ }^{2}$ E Towner, ${ }^{1}$ SR Mashreky, ${ }^{1}$ A Rahman, ${ }^{1}$ A Biswas, ${ }^{1}$ A K M F Rahman. ${ }^{1}$ Centre for Injury Prevention and Research, Bangladesh (CIPRB), Bangladesh;

${ }^{2}$ University of the West of England, UK

Introduction In developing countries, people have social and behavioural misperceptions about injury deaths. Social Autopsy is a process of examining social errors related to injury deaths to determine why they occurred and how they could be prevented by increasing awareness in the community.

Objective To utilise social autopsy for identifying achievable measures to prevent road traffic injury deaths.

Target Audience of Social Autopsies Decision makers in the family, Caretaker of the children, Local leaders.

Principles of Social Autopsy:

- Analyse socially correct behaviours and errors

- Provide positive reinforcement for appropriate behaviours

- Active community participation

- Constructive, supportive facilitation

- Lack of victim blaming

- Conduct immediately after the death

Methods Seventy-seven social autopsies for road traffic injury deaths were conducted in Raiganj, Bangladesh from 2006-2010. About 4000 villagers attended the autopsies. At the start the deceased's family member(s) described the event to the target audience; the facilitator initiated the discussion using standardised protocols and expanded, encouraged and kept the discussion on track to explore the social errors. Recommendations and decisions were made for injury prevention.

Recommendations - Children should be supervised during road use

- Children should not play on the roadside

- Children should be informed about road use from school and family.

- Pedestrians should take care while crossing the road

- Nobody should travel on 'Nosimon' (locally-made unauthorised vehicle)

Conclusions Social Autopsy is a process through which social errors related to the road traffic injury deaths can be elicited and appropriate preventive measures can be identified. 